\title{
GRAPHS WITH LARGE SEMIPAIRED DOMINATION NUMBER
}

\author{
Teresa W. HAYNeS \\ Department of Mathematics and Statistics \\ East Tennessee State University \\ Johnson City, TN 37614-0002 USA \\ and \\ Department of Pure and Applied Mathematics \\ University of Johannesburg \\ Auckland Park 2006, South Africa \\ e-mail: haynes@etsu.edu \\ AND \\ Michael A. Henning \\ Department of Pure and Applied Mathematics \\ University of Johannesburg \\ Auckland Park 2006, South Africa \\ e-mail: mahenning@uj.ac.za
}

\begin{abstract}
Let $G$ be a graph with vertex set $V$ and no isolated vertices. A subset $S \subseteq V$ is a semipaired dominating set of $G$ if every vertex in $V \backslash S$ is adjacent to a vertex in $S$ and $S$ can be partitioned into two element subsets such that the vertices in each subset are at most distance two apart. The semipaired domination number $\gamma_{\mathrm{pr} 2}(G)$ is the minimum cardinality of a semipaired dominating set of $G$. We show that if $G$ is a connected graph $G$ of order $n \geq 3$, then $\gamma_{\mathrm{pr} 2}(G) \leq \frac{2}{3} n$, and we characterize the extremal graphs achieving equality in the bound.
\end{abstract}

Keywords: paired-domination, semipaired domination.

2010 Mathematics Subject Classification: 05C69.

\section{REFERENCES}

[1] W.J. Desormeaux and M.A. Henning, Paired domination in graphs: A survey and recent results, Util. Math. 94 (2014) 101-166. 
[2] W. Goddard, M.A. Henning and C.A. McPillan, Semitotal domination in graphs, Util. Math. 94 (2014) 67-81.

[3] T.W. Haynes, S.T. Hedetniemi and P.J. Slater, Fundamentals of Domination in Graphs (Marcel Dekker, Inc., New York, 1998).

[4] T.W. Haynes, S.T. Hedetniemi and P.J. Slater, Domination in Graphs: Advanced Topics (Marcel Dekker, Inc. New York, 1998).

[5] T.W. Haynes and M.A. Henning, Semipaired domination in graphs, J. Combin. Math. Combin. Comput. 104 (2018) 93-109.

[6] T.W. Haynes and P.J. Slater, Paired-domination and the paired-domatic-number, Congr. Numer. 109 (1995) 65-72.

[7] T.W. Haynes and P.J. Slater, Paired domination in graphs Networks 32 (1998) 199-206. doi:10.1002/(SICI)1097-0037(199810)32:3〈199::AID-NET4〉3.0.CO;2-F

[8] M.A. Henning, Graphs with large paired-domination number, J. Comb. Optim. 13 (2007) 61-78. doi:10.1007/s10878-006-9014-8

[9] M.A. Henning, Edge weighting functions on semitotal dominating sets, Graphs Combin. 33 (2017) 403-417. doi:10.1007/s00373-017-1769-4

[10] M.A. Henning and A.J. Marcon, On matching and semitotal domination in graphs, Discrete Math. 324 (2014) 13-18. doi:10.1016/j.disc.2014.01.021

[11] M.A. Henning and A.J. Marcon, Vertices contained in all or in no minimum semitotal dominating set of a tree, Discuss. Math. Graph Theory 36 (2016) 71-93. doi:10.7151/dmgt.1844

[12] M.A. Henning and A.J. Marcon, Semitotal domination in claw-free cubic graphs, Ann. Comb. 20 (2016) 799-813. doi:10.1007/s00026-016-0331-z

[13] M.A. Henning and A. Yeo, Total Domination in Graphs (Springer Monographs in Mathematics, 2013) doi:10.1007/978-1-4614-6525-6

[14] S. Huang and E. Shan, A note on the upper bound for the paired-domination number of a graph with minimum degree at least two, Networks 57 (2011) 115-116. doi:10.1002/net.20390

Received 7 August 2017 Revised 24 March 2018 Accepted 3 April 2018 